\title{
Research on Instantaneous Dynamical Deformation Monitoring the Masonry Structure Based on Artificial Earthquake Motion
}

\author{
Yongqian $\mathrm{Zhao}^{1,2}$, Yaohua $\mathrm{Wu}^{1}$ and Chengxin $\mathrm{Yu}^{3}$ \\ ${ }^{1}$ School of Control Science and Engineering, Shandong University, Jinan \\ 250061, China \\ ${ }^{2}$ School of Natural Science, Shandong Jianzhu University, Jinan 250101, \\ China \\ ${ }^{3}$ Assets Department, Shandong Jianzhu University, Jinan 250101, China \\ 13455111419@163.com,Mike.Wu@263.net,ycx1108@126.com
}

\begin{abstract}
First, we describe some properties of the masonry structures. It is an urgent task to ensure the safety of the masonry structure by using information technology to monitor the instantaneous deformation dynamically and find out clues of potential hazard real time. This essay gives an empirical research with three-dimensional motion parallax (3-DMP) to this problem, besides; the grey theory model is used to analyze the deformation. The Artificial Earthquake Motional Model (AEM) and the developed software for static and dynamic analyses of different types of masonry structures are illustrated. The combination of multi-technology methods can overcome the error, and play an important role in improving the safety and reliability of the masonry structure. With the analyzed and processed data from digital cameras effectively, we get a precision result approximately equal $2.1 \%$.
\end{abstract}

Keywords: three dimensional motion parallax (3-DMP), the masonry structure, monitor, artificial earthquake motional model

\section{Introduction}

Masonry structures, especially masonry buildings, are probably the most numerous in the history of architecture. One of their main advantages is simple and quick construction [1]. Performed with precast masonry units, bounded by mortar, the brickwork units are usually baked clay, concrete, stone and so on. With different geometrical and physical properties, the horizontal and vertical seam joints between the masonry units are full of mortar. Various types of mortar like lime, lime-cement and cement are used, with different thickness of mortar joints and material properties. The quality of the masonry structures is deeply affected by its compositions-the masonry units, mortar and also the construction quality. Affected by the quality of the bonds between the masonry unit and mortar and the extent of transfer of normal and shear stresses in the surface, the masonry wall has limited strength capacity and deformability (Figure1, 2).

The contribution of non-destructive and instrumental investigation methods is currently exploited to measure and check the evolution of some negative structural phenomena, such as damage and cracking, and to predict their subsequent developments. To choose the technique for controlling and monitoring reinforced masonry structures is strictly related to the type of the structure to be analyzed and on the data to be extracted. The non-destructive method based on Acoustic Emission is proved to be very effective [2, $3]$. This technique makes it possible to estimate the amount of energy released during the fracture process and to obtain information on the durability performances of the monitored structures $[4,5]$. Different from the above, we will make the deformation 
experiments by destructive means and the developed software for static and dynamic analyses of different types of masonry structures.

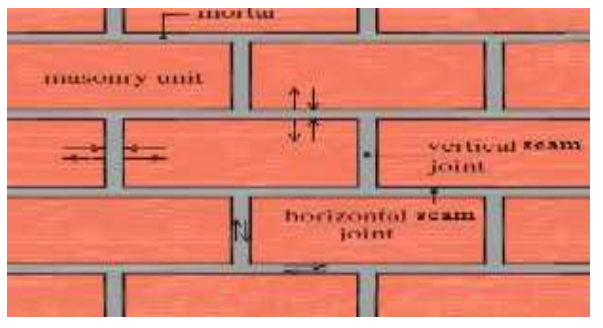

Figure 1. The Typical Masonry Structure

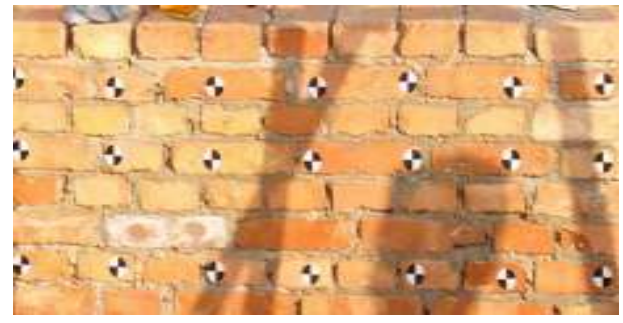

Figure 2. The Masonry Structure for Experiment

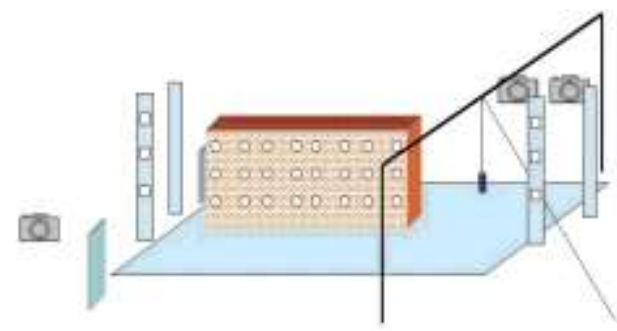

1018

Figure 3. Overall Scene of AEM Model

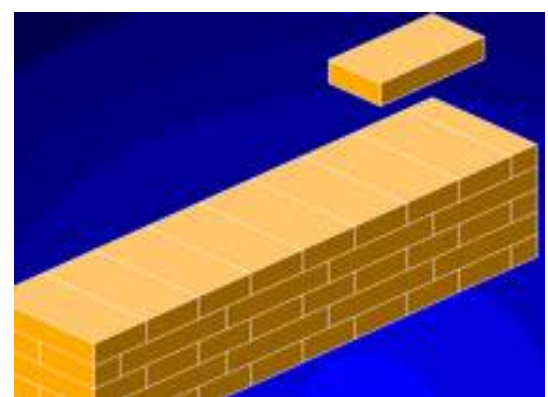

Figure 4. Brick Wall with Yishun Yiding Style

Deformation monitor and analysis are very important for the safety of the masonry buildings. To avoid the loss and safety hazard caused by deformation of the masonry buildings, we should make a feasible solution to monitor the masonry buildings dynamically and continuously. 3-DMP model is used to monitor the masonry structures' deformation in the experiment. Systematically integrated digital cameras and computer to obtain, store, process, manage data and execute other duties, we can process the data with the speed double times. The image is stored stably and fixed color, which has the advantages of non-treatment, automation, real time and so on [6]. In the experiment, we take photos for masonry structure monitored continuously by six digital cameras, the 
images are transported into computer and processed automatically, and the results will be displayed on screen, then we get the deformation details in time. For the 3-DMP model can be used to monitor deformation of many other structures successfully [7], and its simple and cheap devices will be helpful to improve the utilization and safety for the vast of buildings, so it will be accepted by scientific workers and researchers.

The following problems are presented:

1. The AEM model (Figure 3) and 3-DMP method are put forward.

2. Combined with the destructive deformation process of the brick masonry structures, the experiment data is collected and dealt with.

3. Using our method, the monitoring precision can be up to $2.1 \%$.

\section{Two Models for Experiments}

\section{1. Artificial Earthquake Motional Model}

According to the practical situation, we develop an Artificial Earthquake Model illustrated as Figure 3. As a new and convenient method for deformation analysis on the brick masonry structures, the model is used to investigate the instantaneous and dynamical deformation results of the experiment.

A pit covered with Manganese steel plate on it is set in a corner of the construction location. After that, we build a brick masonry wall by YiShun yiding style (See Figure 4) with 1.25 meters long, 1 meter high and 24 centimeters thick bound by mortar on the steel plate. Then set an 8 meter height steel-pipe triangle bracket out of the steel plate distance of 60 centimeters at the south and north directions, through which we hang the customized hammer to impact the plate.

After that, we set four square wood poles at the east and west directions 1 meter off the steel plate. We paste two or three reference marks each on the four poles. On the south and north surface of the brick masonry structure, we set five rows multiple seven columns deformation marks each. On the east and west surface of the brick masonry structure we set six reference marks each.

We use six digital cameras to take photos from four directions for four surfaces of the masonry structure at the same time, and then we get a series of images which records entire continuous deformation process of the masonry structure under the effect of different load.

As a convenient method for deformation analysis, grey theory model is used to predict the results of the experiment. By using GM $(1,1)$, we get some satisfactory results with a little data information. According to the grey system theory [8], we have GM model:

$$
\text { GM }(\mathbf{1}, \mathbf{1}): x_{0}(k)+a z_{1}(k)=b
$$

In which, $x_{1}(k)=\sum_{l=1}^{k} x_{0}(l), z_{1}(k)=\frac{x_{1}(k)+x_{1}(k-1)}{2}, k=2,3, \cdots, n$, and $a, b$ can be

c

$$
\mathrm{m} \quad \mathrm{p} \text { u }
$$

$\mathrm{u}=\mathrm{t}$

$\mathrm{e}$

by $(a, b)^{T}=\left(B^{T} B\right)^{-1} B^{T} Y$, and we have:

$$
Y=\left[\begin{array}{c}
x_{0}(2) \\
x_{0}(3) \\
\vdots \\
x_{0}(n)
\end{array}\right], B=\left[\begin{array}{cc}
-z_{1}(2) & 1 \\
-z_{1}(3) & 1 \\
\vdots & \vdots \\
-z_{1}(n) & 1
\end{array}\right] .
$$

The grey differential equation is $\frac{d x_{1}}{d t}+a x_{1}=b$, so we have:

$$
\hat{x}_{1}(k+1)=\left(x_{1}(0)-\frac{b}{a}\right) e^{-a k}+\frac{b}{a}, \quad k=1,2, \cdots, n
$$


In which:

$$
\hat{x}_{0}(k+1)=\hat{x}_{1}(k+1)-\hat{x}_{1}(k), \quad k=1,2, \cdots, n
$$

\subsection{3-DMP Method}

Different from the typical two dimensional motion parallax to measure two dimensional displacements, 3-DMP model for adjusting digital camera is a method can establish the stereo space relation between coordinate and space position of observation points [9]. Deal with the difference between internal-external azimuths of photos, and adjust its distortion errors. We establish the mathematical model for structural deformation monitoring experiment. In case of given conditions, we can get following solvable mathematical model:

$$
\left\{\begin{array}{l}
x-\Delta x=\frac{a_{1}\left(X-X_{s}\right)+b_{1}\left(Y-Y_{s}\right)+c_{1}\left(Z-Z_{s}\right)}{a_{2}\left(X-X_{s}\right)+b_{2}\left(Y-Y_{s}\right)+c_{2}\left(Z-Z_{s}\right)} \\
z-\Delta z=\frac{a_{3}\left(X-X_{s}\right)+b_{3}\left(Y-Y_{s}\right)+c_{3}\left(Z-Z_{s}\right)}{a_{2}\left(X-X_{s}\right)+b_{2}\left(Y-Y_{s}\right)+c_{2}\left(Z-Z_{s}\right)}
\end{array}\right.
$$

In the upper formula, $x$ and $z$ are the observations of the image points coordinates, $X, Y, Z$ are the ground coordinates, $\Delta x, \Delta z$ are the system error modification values of the image points, $X_{s}, Y_{s}, Z_{s}$ are the ground coordinates of the photography station, $a_{i}, b_{i}, c_{i}(i=1,2,3)$ are the functions of the exterior orientation angle elements.

From formula (5), we have:

$$
\left[\begin{array}{l}
v_{x} \\
v_{z}
\end{array}\right]-\left[\begin{array}{l}
\Delta x \\
\Delta z
\end{array}\right]=\left[\begin{array}{lll}
a_{11} & a_{12} & a_{13} \\
a_{21} & a_{22} & a_{23}
\end{array}\right] \cdot\left[\begin{array}{l}
\delta X \\
\delta Y \\
\delta Z
\end{array}\right]+\left[\begin{array}{cc}
-a_{11} & -a_{21} \\
-a_{12} & -a_{22} \\
-a_{13} & -a_{23} \\
a_{14} & a_{24} \\
a_{15} & a_{25} \\
a_{16} & a_{26}
\end{array}\right]^{T}\left[\begin{array}{l}
\delta X_{s} \\
\delta Y_{s} \\
\delta Z_{s} \\
\delta \varphi \\
\delta \omega \\
\delta \kappa
\end{array}\right]-\left[\begin{array}{l}
x-x_{0} \\
z-z_{0}
\end{array}\right]
$$

In the upper formula, $a_{i j}(i=1,2, \cdots, 6)$ are the coefficients, they are the functions of the principal distance of camera, the ground coordinates, the exterior orientation angle elements and the image point's coordinates. And $x_{0}, z_{0}$ are the coordinates of the image points.

Let header label "I"denote the content of the phase "I", and the "II" denote the content of the phase "II", and let $\delta X=(\delta x, \delta y, \delta z)^{T}, \delta X_{s}=\left(\delta X_{s}, \delta Y_{s}, \delta Z_{s}, \delta \varphi, \delta \omega, \delta k\right)^{T}$, then we can obtain the error equations of the two phase image point coordinates:

$$
\left[\begin{array}{c}
v_{x}^{I} \\
v_{z}^{I}
\end{array}\right]-\left[\begin{array}{c}
\Delta^{I} x \\
\Delta^{I} z
\end{array}\right]=\left[\begin{array}{lll}
a_{11}^{I} & a_{12}^{I} & a_{13}^{I} \\
a_{21}^{I} & a_{22}^{I} & a_{23}^{I}
\end{array}\right] \delta X^{I}+\left[\begin{array}{cc}
-a_{11}^{I} & -a_{21}^{I} \\
-a_{12}^{I} & -a_{22}^{I} \\
-a_{13}^{I} & -a_{23}^{I} \\
a_{14}^{I} & a_{24}^{I} \\
a_{15}^{I} & a_{25}^{I} \\
a_{16}^{I} & a_{26}^{I}
\end{array}\right]^{T} \delta X^{I}{ }_{s}-\left[\begin{array}{c}
x^{I}-x_{0}^{I} \\
z^{I}-z_{0}^{I}
\end{array}\right]
$$




$$
\left[\begin{array}{l}
v_{x}^{I I} \\
v_{z}^{I I}
\end{array}\right]-\left[\begin{array}{l}
\Delta^{I I} x \\
\Delta^{I I} z
\end{array}\right]=\left[\begin{array}{lll}
a_{11}^{I I} & a_{12}^{I I} & a_{13}^{I I} \\
a_{21}^{I I} & a_{22}^{I I} & a_{23}^{I I}
\end{array}\right] \delta X^{I I}+\left[\begin{array}{cc}
-a_{11}^{I I} & -a_{21}^{I I} \\
-a_{12}^{I I} & -a_{22}^{I I} \\
-a_{13}^{I I} & -a_{23}^{I I} \\
a_{14}^{I I} & a_{24}^{I I} \\
a_{15}^{I I} & a_{25}^{I I} \\
a_{16}^{I I} & a_{26}^{I I}
\end{array}\right]^{T} \delta X^{I I}{ }_{s}-\left[\begin{array}{c}
x^{I I}-x_{0}^{I I} \\
z^{I I}-z_{0}^{I I}
\end{array}\right]
$$

then we can obtain formula $(9,10)$ based on the parallax definition:

Horizontal direction $p=\left(x^{I}-\Delta x^{I}\right)-\left(x^{I I}-\Delta x^{I I}\right)=\left(x^{I}-x^{I I}\right)-\left(\Delta x^{I}-\Delta x^{I I}\right)$

Vertical direction $q=\left(z^{I}-\Delta z^{I}\right)-\left(z^{I I}-\Delta z^{I I}\right)=\left(z^{I}-z^{I I}\right)-\left(\Delta z^{I}-\Delta z^{I I}\right)$

Consider $\Delta x^{I} \approx \Delta x^{I I}, \Delta z^{I} \approx \Delta z^{I I}$ and we can obtain (11):

$$
\left\{\begin{array}{l}
p=x^{I}-x^{I I} \\
q=z^{I}-z^{I I}
\end{array}\right.
$$

Suppose $a_{i j}^{I} \approx a_{i j}^{I I}=a_{i j}, x_{0}^{I}=x_{0}^{I I}, z_{0}^{I}=z_{0}^{I I}, \delta x=\delta x^{I}-\delta x^{I I}, \delta x_{s}=\delta x_{s}^{I}-\delta x_{s}^{I I}$, and formula (7) minus formula (8), we

$$
\begin{gathered}
\text { have: }\left[\begin{array}{l}
U_{p} \\
U_{q}
\end{array}\right]=\left[\begin{array}{lll}
a_{11} & a_{12} & a_{13} \\
a_{21} & a_{22} & a_{23}
\end{array}\right] \delta X+\left[\begin{array}{llllll}
-a_{11} & -a_{12} & -a_{13} & a_{14} & a_{15} & a_{16} \\
-a_{21} & -a_{22} & -a_{23} & a_{24} & a_{25} & a_{26}
\end{array}\right] \delta X_{s}-\left[\begin{array}{l}
p \\
q
\end{array}\right] \\
V=A \delta X+B \delta X_{s}-L \\
V=A
\end{gathered}
$$

\section{The Monitoring Experiment on the Masonry Structure}

In our experiment, we use six digital cameras to take sequence photos on the dynamic changing masonry structure. Then transmit real time images into computers, process the data of images automatically and obtain the monitor data in the field.

\subsection{Several Calculating Results}

When we let the hammer impact the steel plate, the masonry structure will do simple harmonic vibration. So we have $x=A \cos (\omega t+\varphi), \omega=\frac{2 \pi}{T}$, and the velocity is:

$$
v=\frac{d x}{d t}=-A \cdot \frac{2 \pi}{T} \cdot \sin \left(\frac{2 \pi}{T} t+\varphi\right)
$$

Accordingly, we can deduce the acceleration of the masonry structure at every impact on the steel plate as follow:

$$
|a|=\left|\frac{d^{2} x}{d t^{2}}\right|=\left|\frac{d}{d t}\left(\frac{d x}{d t}\right)\right|=\left|\frac{d}{d t}\left[A \cdot \frac{2 \pi}{T} \cdot \sin \left(\frac{2 \pi}{T} t+\varphi\right)\right]\right|=|A| \cdot\left|\frac{4 \pi^{2}}{T^{2}} \cos \left(\frac{2 \pi}{T} t+\varphi\right)\right| \leq \frac{4 \pi^{2} \cdot|A|}{T^{2}}
$$

The acceleration can be computed in Excel 2007; the results are in Table 1.

\subsection{Details of the Monitoring Process}

\subsubsection{Overall Design}

It is a proper choose to make a construction site as experiment field when it is in the foundation treatment stage. At the site we choose, the soil is the native yellow silt clay 
with high compressive strength. The overall design of the artificial earthquake model is illustrated as Figure 3.

\subsubsection{Set Marks}

Before the experiment, we will paste enough artificial deformation marks and reference marks onto the brick masonry wall and reference pole. We can get the deformation state of the masonry wall by processing the images. Number and record every point for the use of their motion state analysis. We set four square wood poles at the east and west directions off the steel plate; every pole has six marks on the North and South surface, two or three reference marks on the East and west surface (See Figure6). At the south and north surface of the brick masonry wall, we set 35 deformation marks and 4 auxiliary deformation marks each. On the east and west surface of the brick masonry structure we set six reference marks each (See Figure 5).

Table 1. The Acceleration Calculating Processes

\begin{tabular}{|c|c|c|c|c|c|c|c|c|c|c|}
\hline \multirow{2}{*}{$\begin{array}{l}\text { test } \\
\text { times }\end{array}$} & \multirow{2}{*}{$\begin{array}{c}\text { height } \\
(m)\end{array}$} & \multirow{2}{*}{$\begin{array}{c}\text { velocity } \\
(\mathrm{m} / \mathrm{s})\end{array}$} & \multirow{2}{*}{$\underset{(\mathrm{kg} \cdot \mathrm{m} / \mathrm{s})}{\operatorname{momentum}}$} & \multicolumn{3}{|c|}{ impact force $(\mathrm{N})$} & \multicolumn{3}{|c|}{ acceleration $\left(\mathrm{m} / \mathrm{s}^{2}\right)$} & \multirow{2}{*}{$\begin{array}{l}\text { actual } \\
\text { settlement } \\
(\mathrm{cm})\end{array}$} \\
\hline & & & & $\mathrm{T}=\mathbf{0 . 0 5}$ & $\mathrm{T}=\mathbf{0 . 1}$ & $\mathrm{T}=\mathbf{0 . 2}$ & $\mathbf{T}=\mathbf{0 . 0 5}$ & $\mathrm{T}=0.1$ & $\mathrm{~T}=0.2$ & \\
\hline 1 & 0.3 & 2.4249 & 60.622 & 1212.436 & 606.218 & 303.109 & 78.957 & 39.478 & 19.739 & 0.1000 \\
\hline 2 & 0.6 & 3.4293 & 85.732 & 1714.643 & 857.321 & 428.661 & 11.054 & 5.527 & 2.763 & 0.0140 \\
\hline 3 & 0.9 & 4.2000 & 105.000 & 2100.000 & 1050.000 & 525.000 & 22.897 & 11.449 & 5.724 & 0.0290 \\
\hline 4 & 1.2 & 4.8497 & 121.244 & 2424.871 & 1212.436 & 606.218 & 101.854 & 50.927 & 25.464 & 0.1290 \\
\hline 5 & 1.5 & 5.4222 & 135.554 & 2711.088 & 1355.544 & 677.772 & -11.054 & -5.527 & -2.763 & -0.0140 \\
\hline 6 & 1.8 & 5.9397 & 148.492 & 2969.848 & 1484.924 & 742.462 & 56.059 & 28.030 & 14.015 & 0.0710 \\
\hline 7 & 2.1 & 6.4156 & 160.390 & 3207.803 & 1603.901 & 801.951 & 11.054 & 5.527 & 2.763 & 0.0140 \\
\hline 8 & 2.4 & 6.8586 & 171.464 & 3429.286 & 1714.643 & 857.321 & 0.000 & 0.000 & 0.000 & 0.0000 \\
\hline 9 & 2.7 & 7.2746 & 181.865 & 3637.307 & 1818.653 & 909.327 & 33.951 & 16.976 & 8.488 & 0.0430 \\
\hline 10 & 3 & 7.6681 & 191.703 & 3834.058 & 1917.029 & 958.514 & 90.011 & 45.005 & 22.503 & 0.1140 \\
\hline 11 & 3.3 & 8.0424 & 201.060 & 4021.194 & 2010.597 & 1005.298 & 33.951 & 16.976 & 8.488 & 0.0430 \\
\hline 12 & 3.6 & 8.4000 & 210.000 & 4200.000 & 2100.000 & 1050.000 & 67.903 & 33.951 & 16.976 & 0.0860 \\
\hline 13 & 3.9 & 8.7430 & 218.575 & 4371.499 & 2185.749 & 1092.875 & 33.951 & 16.976 & 8.488 & 0.0430 \\
\hline 14 & 4.2 & 9.0730 & 226.826 & 4536.518 & 2268.259 & 1134.130 & -11.054 & -5.527 & -2.763 & -0.0140 \\
\hline 15 & 4.5 & 9.3915 & 234.787 & 4695.743 & 2347.871 & 1173.936 & 22.897 & 11.449 & 5.724 & 0.0290 \\
\hline 16 & 4.8 & 9.6995 & 242.487 & 4849.742 & 2424.871 & 1212.436 & 101.854 & 50.927 & 25.464 & 0.1290 \\
\hline 17 & 5.1 & 9.9980 & 249.950 & 4999.000 & 2499.500 & 1249.750 & 45.005 & 22.503 & 11.251 & 0.0570 \\
\hline 18 & 5.4 & 10.2879 & 257.196 & 5143.928 & 2571.964 & 1285.982 & 78.957 & 39.478 & 19.739 & 0.1000 \\
\hline 19 & 5.4 & 10.2879 & 257.196 & 5143.928 & 2571.964 & 1285.982 & 22.897 & 11.449 & 5.724 & 0.0290 \\
\hline & $\begin{array}{l}\text { Out } \\
\text { Opr }\end{array}$ & ve & uno & $20 \mathrm{iga}$ & r & -2 & $\begin{array}{l}\square^{*} \\
\square^{*} \\
\Xi^{*}\end{array}$ & $\begin{array}{l}\square^{*} \\
\square * \\
\square * \\
\square\end{array}$ & $|c|$ & \\
\hline & (a) $\mathrm{N}$ & orth S & & (b) & ast $\mathrm{Si}$ & & Soutl & & 8 & ide \\
\hline
\end{tabular}

Figure 5. Number of the Deformation and Reference Marks on the Masonry Structure and Poles 


\subsubsection{Distance Measuring}

By measuring the distance between every two points above the marks and the photography position, we get the location relations of all experiment components. Number and record every point for the use of their motion state analysis (See Figure 5).

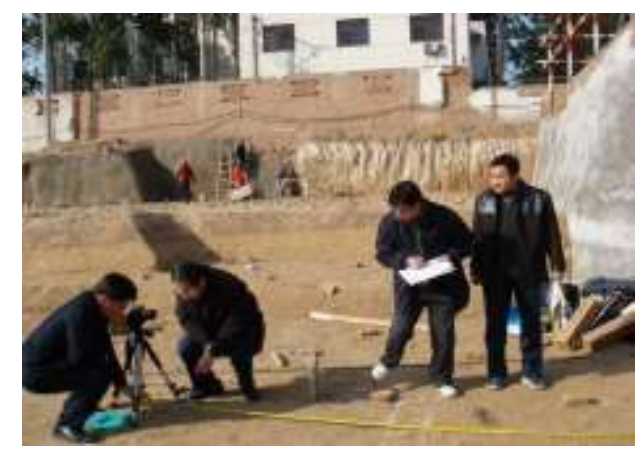

(a)Distance of the Photography Position

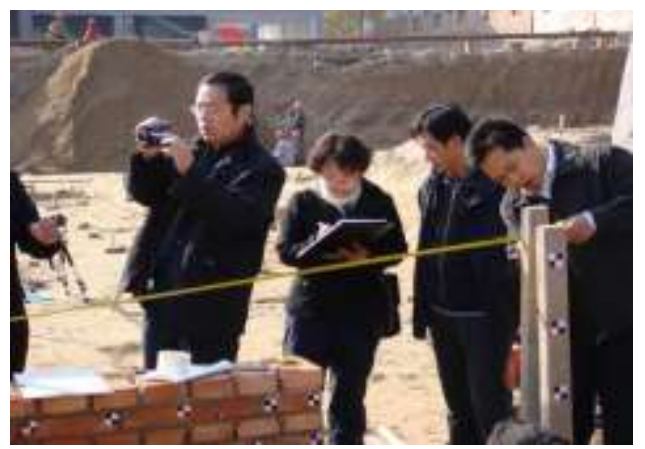

(b) Distance of the Base Line

Figure 6. Measure the Distance

\subsubsection{Take Photos}

Firstly, we take photos by every camera simultaneously as the zero(original) photos. Then lift the hammer to a certain height safety and stability, and let it fall freely in different height until the masonry structure destroyed. We use six digital cameras to take photos from four directions simultaneously at the impact moment when the hammer falls from every certain height.

\subsubsection{Image Processing}

Firstly, put every camera`s photo into a folder and transfer the series of images to ".bmp" format. Open the BMP bitmap file, measure the point coordinates of reference points and the deformation points on every image, and the program we developed will automatically keep the pixel location measurement data of the reference points and deformation points at the end of the BMP bitmap file directly. When finished the measure stage, open the BMP bitmap file again one by one and input the given load and distance data, the deformation status curve will be displayed on the computer's screen.

\section{Several Cartographic Data}

\subsection{Measured Tables}

Measure the relative distance between two points above the deformation marks and reference marks and the photography position, up to the accuracy $0.1 \mathrm{~mm}$. Table $2-$ Table 4 show the overall of the distance we measured before experiment.

\subsection{Pixel Coordinates}

Table 5 show partial data of the pixel coordinates of reference and deformation points when the hammer falls from the height $0.3 \mathrm{~m}, 3.9 \mathrm{~m}, 4.2 \mathrm{~m}, 4.5 \mathrm{~m}, 4.8 \mathrm{~m}, 5.1 \mathrm{~m}$ and $5.4 \mathrm{~m}$.

\subsection{Partial Deformation Curve Maps}

From the above data and images we can obtain the deformation curve contrast maps of $\mathrm{u} 0-\mathrm{u} 5$ and some other deformation points as Figure 7 shows. 
Table 2. Vertical Distance between Every Two Deformation Points (North Side)

\begin{tabular}{c|c|c|c|c|c|c|c}
\hline $\begin{array}{c}\text { point } \\
\text { marks }\end{array}$ & $\begin{array}{c}\text { distance } \\
\text { cm) }\end{array}$ & $\begin{array}{c}\text { point } \\
\text { marks }\end{array}$ & $\begin{array}{c}\text { distance } \\
\mathbf{c m})\end{array}$ & $\begin{array}{c}\text { point } \\
\text { marks }\end{array}$ & $\begin{array}{c}\text { distance } \\
\text { cm) }\end{array}$ & $\begin{array}{c}\text { point } \\
\text { marks }\end{array}$ & $\begin{array}{c}\text { distance } \\
\text { cm) }\end{array}$ \\
\hline $\mathrm{u} 0-\mathrm{u} 1$ & 13.2 & $\mathrm{u} 8-\mathrm{u} 9$ & 17.1 & $\mathrm{u} 17-\mathrm{u} 18$ & 20.2 & $\mathrm{u} 26-\mathrm{u} 27$ & 19.3 \\
\hline $\mathrm{u} 1-\mathrm{u} 2$ & 17.9 & $\mathrm{u} 10-\mathrm{u} 11$ & 12.2 & $\mathrm{u} 18-\mathrm{u} 19$ & 17.2 & $\mathrm{u} 27-\mathrm{u} 28$ & 18.6 \\
\hline $\mathrm{u} 2-\mathrm{u} 3$ & 19.7 & $\mathrm{u} 11-\mathrm{u} 12$ & 19.4 & $\mathrm{u} 20-\mathrm{u} 21$ & 12.4 & $\mathrm{u} 28-\mathrm{u} 29$ & 18.0 \\
\hline $\mathrm{u} 3-\mathrm{u} 4$ & 17.4 & $\mathrm{u} 12-\mathrm{u} 13$ & 20.0 & $\mathrm{u} 21-\mathrm{u} 22$ & 19.4 & $\mathrm{u} 30-\mathrm{u} 31$ & 11.5 \\
\hline $\mathrm{u} 5-\mathrm{u} 6$ & 12.7 & $\mathrm{u} 13-\mathrm{u} 14$ & 19.8 & $\mathrm{u} 22-\mathrm{u} 23$ & 19.6 & $\mathrm{u} 31-\mathrm{u} 32$ & 19.8 \\
\hline $\mathrm{u} 6-\mathrm{u} 7$ & 18.6 & $\mathrm{u} 15-\mathrm{u} 16$ & 12.2 & $\mathrm{u} 23-\mathrm{u} 24$ & 16.7 & $\mathrm{u} 32-\mathrm{u} 33$ & 19.6 \\
\hline $\mathrm{u} 7-\mathrm{u} 8$ & 19.2 & $\mathrm{u} 16-\mathrm{u} 17$ & 19.2 & $\mathrm{u} 25-\mathrm{u} 26$ & 12.7 & $\mathrm{u} 33-\mathrm{u} 34$ & 18.0 \\
\hline
\end{tabular}

Table 3. Horizontal Distance between Every Two Deformation Points (North Side)

\begin{tabular}{c|c|c|c|c|c|c|c|}
\hline $\begin{array}{c}\text { point } \\
\text { marks }\end{array}$ & $\begin{array}{c}\text { distance } \\
\mathbf{c m})\end{array}$ & $\begin{array}{c}\text { point } \\
\text { marks }\end{array}$ & $\begin{array}{c}\text { distance } \\
\text { cm) }\end{array}$ & $\begin{array}{c}\text { point } \\
\text { marks }\end{array}$ & $\begin{array}{c}\text { distance } \\
\text { cm) }\end{array}$ & $\begin{array}{c}\text { point } \\
\text { marks }\end{array}$ & $\begin{array}{c}\text { distance } \\
\text { cm) }\end{array}$ \\
\hline $\mathrm{u} 0-\mathrm{u} 5$ & 18.5 & $\mathrm{u} 25-\mathrm{u} 30$ & 12.0 & $\mathrm{u} 3-\mathrm{u} 8$ & 17.1 & $\mathrm{u} 19-\mathrm{u} 24$ & 18.8 \\
\hline $\mathrm{u} 5-\mathrm{u} 10$ & 20.2 & $\mathrm{u} 1-\mathrm{u} 6$ & 17.6 & $\mathrm{u} 8-\mathrm{u} 3$ & 19.6 & $\mathrm{u} 24-\mathrm{u} 29$ & 21.8 \\
\hline $\mathrm{u} 10-\mathrm{u} 15$ & 20.3 & $\mathrm{u} 6-\mathrm{u} 11$ & 20.0 & $\mathrm{u} 4-\mathrm{u} 9$ & 17.6 & $\mathrm{u} 29-\mathrm{u} 34$ & 16.5 \\
\hline $\mathrm{u} 15-\mathrm{u} 20$ & 18.9 & $\mathrm{u} 2-\mathrm{u} 7$ & 17.5 & $\mathrm{u} 9-\mathrm{u} 14$ & 19.8 & & \\
\hline $\mathrm{u} 20-\mathrm{u} 25$ & 23.7 & $\mathrm{u} 7-\mathrm{u} 12$ & 19.8 & $\mathrm{u} 14-\mathrm{u} 19$ & 19.3 & & \\
\hline
\end{tabular}

Table 4. Actual Distance and the Pixel Coordinate Distance Ratio Table

\begin{tabular}{c|c|c|c}
\hline $\begin{array}{c}\text { point } \\
\text { marks }\end{array}$ & $\begin{array}{c}\text { actual distance } \\
(\mathbf{c m})\end{array}$ & $\begin{array}{c}\text { pixel coordinate } \\
\text { distance(cm) }\end{array}$ & $\begin{array}{c}\text { ratio } \\
(\mathbf{p i x} / \mathbf{c m})\end{array}$ \\
\hline $\mathrm{C} 0 \mathrm{C} 1$ & 20.8 & 151 & 7.260 \\
\hline $\mathrm{C} 1 \mathrm{C} 2$ & 21.1 & 155 & 7.346 \\
\hline $\mathrm{C} 2 \mathrm{C} 3$ & 20.4 & 150.4 & 7.373 \\
\hline $\mathrm{C} 3 \mathrm{C} 4$ & 19.3 & 142.4 & 7.378 \\
\hline $\mathrm{C} 6 \mathrm{C} 7$ & 15.6 & 104.6 & 6.705 \\
\hline $\mathrm{C} 7 \mathrm{C} 8$ & 17.2 & 116.8 & 6.791 \\
\hline C8C9 & 20.5 & 136.6 & 6.663 \\
\hline Average & 19.271 & 136.686 & 7.093 \\
\hline
\end{tabular}

Table 5. Pixel Coordinates of Reference and Deformation Points (Partial Data )

\begin{tabular}{c|c|c|c|c|c|c|c|c|c|c|c|c|c|c}
\hline \multirow{2}{*}{$\begin{array}{c}\text { Load } \\
(\mathbf{2 5 k g})\end{array}$} & \multicolumn{2}{|c|}{$\mathbf{0 . 3 m}$} & \multicolumn{2}{c|}{$\mathbf{3 . 9 m}$} & \multicolumn{2}{c|}{$\mathbf{4 . 2 m}$} & \multicolumn{2}{c|}{$\mathbf{4 . 5 m}$} & \multicolumn{2}{|c|}{$\mathbf{4 . 8 m}$} & \multicolumn{2}{|c|}{$\mathbf{5 . 1 m}$} & \multicolumn{2}{|c}{$\mathbf{5 . 4 m}$} \\
\cline { 2 - 15 } & $\mathbf{X}$ & $\mathbf{Y}$ & $\mathbf{X}$ & $\mathbf{Y}$ & $\mathbf{X}$ & $\mathbf{Y}$ & $\mathbf{X}$ & $\mathbf{Y}$ & $\mathbf{X}$ & $\mathbf{Y}$ & $\mathbf{X}$ & $\mathbf{Y}$ & $\mathbf{X}$ & $\mathbf{Y}$ \\
\hline $\mathrm{C}_{0}$ & 962 & 1374 & 962 & 1376 & 961 & 1375 & 962 & 1374 & 1026 & 1375 & 1027 & 1375 & 961 & 1373 \\
\hline $\mathrm{C}_{1}$ & 966 & 1525 & 966 & 1528 & 965 & 1527 & 965 & 1525 & 1031 & 1525 & 1030 & 1528 & 965 & 1524 \\
\hline $\mathrm{C}_{2}$ & 968 & 1680 & 967 & 1683 & 967 & 1681 & 967 & 1680 & 1033 & 1680 & 1033 & 1683 & 968 & 1679 \\
\hline $\mathrm{C}_{3}$ & 969 & 1831 & 968 & 1833 & 968 & 1832 & 968 & 1831 & 1034 & 1831 & 1033 & 1833 & 968 & 1829 \\
\hline $\mathrm{C}_{4}$ & 974 & 1973 & 974 & 1975 & 973 & 1975 & 975 & 1973 & 1038 & 1974 & 1039 & 1975 & 974 & 1972 \\
\hline $\mathrm{C}_{5}$ & 979 & 2185 & 977 & 2187 & 977 & 2187 & 977 & 2184 & 1043 & 2185 & 1042 & 2187 & 977 & 2183 \\
\hline $\mathrm{U}_{0}$ & 2353 & 1663 & 2353 & 1668 & 2353 & 1666 & 2355 & 1666 & 2420 & 1668 & 2423 & 1669 & 2358 & 1667 \\
\hline $\mathrm{U}_{1}$ & 2355 & 1751 & 2355 & 1756 & 2354 & 1755 & 2356 & 1755 & 2421 & 1756 & 2422 & 1757 & 2357 & 1756 \\
\hline $\mathrm{U}_{2}$ & 2358 & 1881 & 2358 & 1887 & 2357 & 1885 & 2359 & 1885 & 2424 & 1886 & 2425 & 1888 & 2361 & 1887 \\
\hline $\mathrm{U}_{3}$ & 2359 & 2017 & 2361 & 2022 & 2360 & 2021 & 2361 & 2020 & 2427 & 2022 & 2429 & 2023 & 2364 & 2021 \\
\hline $\mathrm{U}_{4}$ & 2360 & 2146 & 2360 & 2152 & 2359 & 2151 & 2360 & 2150 & 2424 & 2152 & 2424 & 2153 & 2360 & 2151 \\
\hline $\mathrm{U}_{5}$ & 2233 & 1668 & 2233 & 1674 & 2233 & 1673 & 2235 & 1672 & 2300 & 1674 & 2301 & 1675 & 2236 & 1674 \\
\hline
\end{tabular}



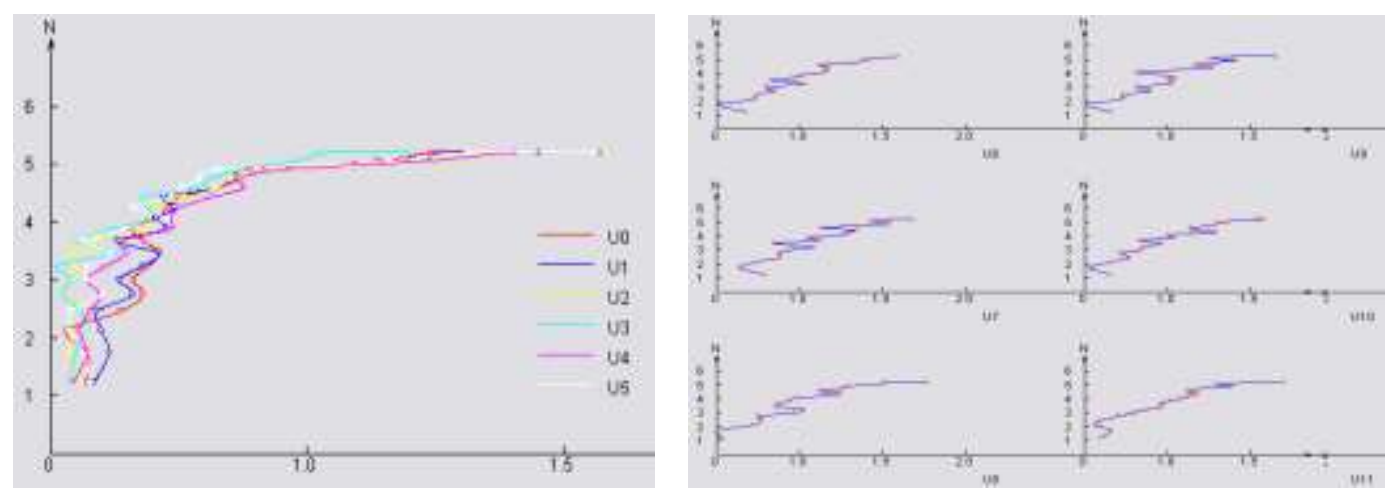

Figure 7. Deformation Curve Maps of Deformation Points

\section{The Conclusions}

The masonry units and mortar's compressive strength determine the transfer of normal compressive stresses on the surface. The strength capacity has a great difference between the horizontal and vertical seam joints. Vertical compressive stresses in masonry are much higher due to the mode of placing and being filled with mortar only partially, the vertical seam joint is usually of less strength than the mortar in horizontal seam joints. The transfer of normal tensile stresses perpendicular to the joints is governed by the adhesion between mortar and masonry unit. The extent of shear transfer in horizontal joints is greater than in vertical joints because of higher quality and better adhesion between the mortar and the masonry unit, especially the favorable vertical compressive stress effect.

The deformation curve maps in Figure 7 reflects the deformation process as the impact force is put on the masonry structure step by step, which is suitable for the deformation principle of the masonry structure. There are so much data collected from the experiment, so we only show the $3^{\text {rd }}$ camera photos' maps of the deformation points which distorted more obvious relatively as displayed.

We can find the most destructive part of the masonry structure is near the impact position of the steel plate, especially the part in the red ellipse, seeing Figure 8. Except that, we can also find damage cracks distribute in cross shape as displayed in red slashes. And also the damage at the bottom is greater than the top.

By using our artificial earthquake model and three-dimensional time baseline parallax method for the deformation monitoring, we can realize the process: data acquisition, processing and graphical display function. Except that, we also can make the operation more convenient, efficient, and the digital cameras can be flexible placed with a certain angle, and the requirement is looser for actual monitoring site, and make it easy to implement a remote monitoring work. By using pixels of more than 4 million digital cameras and data processing system, we can do measurement work in 6 meters. The accuracy is approximately $2.1 \%$, which can satisfy the requirements of the precision of monitoring the masonry structure vibration deformation. This system can also be used for monitoring other types of building structure deformation dynamically [10]. 


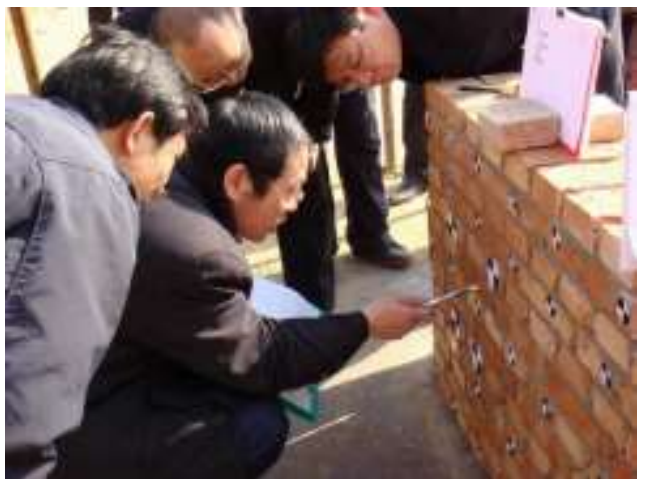

(a) Observation the Deformation Status

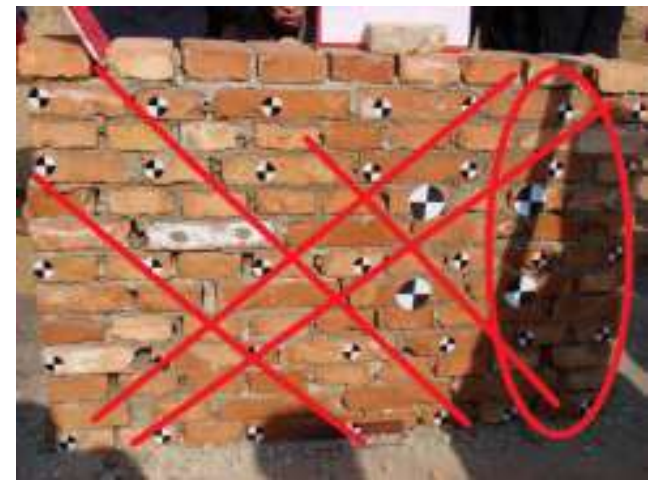

(b) The Destroyed Masonry Structure

Figure 8 Actual Deformation Status of the Masonry Structure

It is difficult to reach or realize real-time monitoring during the construction process of the machinery and buildings [11]. By using our method and the program, one can install the cameras with various higher performance indicators near the scene without any dangerous accidents.

\section{ACKNOWLEDGEMENTS}

Project supported by the National Nature Science Foundation of China (No. 61304008), Shandong Province Major Scientific Research Projects (2010GX20125), and Doctor's Foundation of Shandong Jianzhu University (XNBS: 1338). Thanks very much to my doctoral supervisor-Professor Yaohua Wu, whose research interests include the designing and simulation for integration logistics system, theories of automated order picking system and logistics equipment, etc. Thanks very much also to my doctoral Cosupervisor-Professor Chengxin Yu, whose research interests include the logistics system engineering, system optimization, and computing science, etc.

\section{References}

[1] J. Radnic, D. Matešan, A. Harapin, M. Smilovic and N. Grgic, "Numerical Model for Static and Dynamic Analysis of Masonry Structures", Mechanics and Properties of Composed Materials and Structures, Springer-Verlag Berlin Heidelberg, vol. 31, (2012), pp. 1-33.

[2] A. Carpinteri and P. Bocca, "Damage and Diagnosis of Materials and Structures", Pitagora Ed., Bologna, Italy, (1991).

[3] A. Carpinteri, G. Lacidogna and N. Pugno, "In Proceedings of 5th International Conference on Fracture Mechanics of Concrete and Concrete Structures", (FraMCos-5), edited by V. C.Li, C. K. Y. Leung, K. J. Willam, S. L. Billington, (2004), pp. 31-40.

[4] A. Carpinteri and G. Lacidogna, "Journal Facta Universitatis, Series: Mechanics", Automatic Control and Robotics, vol. 19, (2003), pp. 755- 764.

[5] A. Carpinteri and G. Lacidogna, "System for the assessment of safety conditions in reinforced concrete and masonry structures", Italian Patent N. To 2002 A000924, deposited on, (2002) October 23.

[6] F. Wenhao, "Close-Range Photogrammetry", Wuhan Technical University of Surveying and Mapping, (2000).

[7] M. Chen, C. Yu, N. Xu and Y. Zhao, "Application Study of Digital Analytical Method on Deformation Monitor of high-rise Goods Shelf", Proceedings of the IEEE International Conference on Automation and Logistics Qingdao, China, (2008) September, pp. 2084-2088.

[8] Y. Zhao, "An application of the grey forecast theory to the deformation analysis", (in Chinese), Shandong Science, vol. 21, no. 2, (2008) April, pp. 42-45.

[9] D. Xinhua, L. Su and W. Huailin, "Application of three-dimensional motion parallax in deformation monitoring of logistic shelves", (in Chinese), Shandong Science, vol.24, no. 4, (2011), pp. 52-57.

[10] Y. Chengxin, "Exploration of the deformation analysis by using gray system theory", (in Chinese), Shandong Jianzhu University Journal, no. 2, (1999).

[11] T. Hara and M. Nakabo, "Dynamic Deformation Measurement of Real Bridge Using Sampling Moiré Camera", Proceedings of the IEEE International Conference on Intelligent Computing and Integrated Systems, (2011).

\section{Authors}




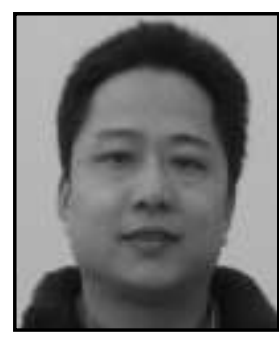

Yongqian Zhao, (1972.07-) He is a PhD candidate student in School of Control Science and Engineering, Shandong University, Jinan, China. And also is an associate professor in the School of Natural Science, Shandong Jianzhu University. His research interests include Systematical Engineering and Logistic optimization.

Email address: 13455111419@163.com .

Tel.: +86 13455111419 
International Journal of Hybrid Information Technology

Vol.8, No.10 (2015) 